Ann. Sci. forest., 1977, 34 (4), 323-329.

\title{
Réflexions sur les premiers résultats d'un dispositif d'éclaircies de pins sylvestres en forêt de Lamotte-Beuvron
}

\author{
Jean BOUCHON \\ Station de Sylviculture ef production, \\ Centre National de Recherches Forestières, I.N.R.A., \\ Champenoux, F 54280 Seichamps
}

\section{Résumé}

II est quelquefois malaisé de mettre en évidence la concurrence en forêt, du fait de la multitude des facteurs qui entrent en jeu. Une expérience a confirmé l'effet important de la densité moyenne du peuplement ; il est encore difficile de se prononcer sur la concurrence entre strates et sur les effets de la densité locale.

\section{1. - Généralités sur la concurrence en forêt}

\section{1. - Définitions}

1.11. La compétition entre 2 organismes.

est le résultat des relations entre ces individus ; l'influence de la compétition sur un organisme peut être positive, négative ou nulle ; on parlera de concurrence lorsque l'influence de la compétition est négative sur les 2 individus : c'est le cas général entre les arbres ; mais Illy et B. Lemoine (1970) d'une part, Decourt et M. Lemoine (1974) d'autre part, ont montré qu'il n'en était pas toujours ainsi dans le jeune âge.

1.12. La structure d'un peuplement.

pour une grandeur quelconque est la distribution spatiale de cette grandeur mesurée sur les arbres du peuplement; on ne confondra pas la structure d'un peuplement avec l'histogramme du nombre de tiges par catégories de diamètre.

\section{2. - La concurrence en forêt}

A un instant donné, les dimensions d'un arbre sont déterminées par toute son histoire ; elles intègrent l'action combinée de facteurs : 
- externes à l'arbre (milieu, concurrence des autres arbres, éclaicies, fertilisation et traitements divers),

— internes à l'arbre (génétique, aptitude à la concurrence, dégâts divers).

D'autre part, les peuplements forestiers sont le plus souvent génétiquement hétérogènes, ce qui provoque une variabilité phénotypique assez grande et une stratification sociologique.

La pérennité et la variabilité des arbres compliquent donc l'analyse ; chaque arbre exerce et subit une concurrence en relation avec la position sociale qu'il a acquise. Par la suite, nous ferons les deux hypothèses simplificatrices suivantes :

a) avec Leary (1970), nous supposerons que la concurrence ne s'exerce qu'à l'intérieur d'une même classe ou d'une classe dominante vers les classes dominées. Mais on a une succession continue de classes et du point de vue de la concurrence, tout se passe comme si on avait un mélange continu d'espèces : on est loin des mélanges à 2 constituants de De Wit (1960) et de Jacquard (1975). On fait donc une deuxième simplification :

b) on supposera que le peuplement se réduit à 2 strates : les dominants et les dominés.

Ces deux hypothèses peuvent ainsi être formulées d'une manière plus simple : dans un peuplement forestier, les gros se concurrencent entre eux et concurrencent les petits ; les petits se concurrencent entre eux et sont concurrencés par les gros. II s'agit bien entendu d'une première approximation, qui est cependant suffisante pour l'expérience qui nous concerne ici.

\section{2. - Mise en évidence de la concurrence : dispositif expérimental}

Pour mettre en évidence la concurrence dans un peuplement, on choisit a priori un certain nombre d'arbres sur lesquels on mesure périodiquement l'accroissement sur le diamètre à $1,30 \mathrm{~m}$, hauteur habituelle de mesure. Sur ces arbres sélectionnés, on admet que la concurrence apparaît de plusieurs manières :

a) par un effet global dû à la densité moyenne du peuplement,

b) par un effet local dû à la densité au voisinage de l'arbre,

c) par un effet de concurrence de la strate dominante vers la strate dominée.

\section{Dispositif imaginé.}

Un peuplement de pin sylvestre planté à $1,5 \mathrm{~m} \times 1,5 \mathrm{~m}$ en 1948 en forêt domaniale de Lamotte-Beuvron (Loiret) a été choisi ; un test d'homogénéité fait sur la hauteur des arbres a conduit à installer un dispositif en carré latin, la fertilité étant plus faible au centre de la parcelle expérimentale ; les hauteurs moyennes de placettes de $30 \mathrm{~m}^{2}$ variant de 5,3 à $7,3 \mathrm{~m}$, la comparaison avec la table de production de Décourt (1965) a permis d'assimiler ce peuplement à la classe de fertilité II ; les mesures de hauteur dominante faites ultérieurement confirment ce classement. 


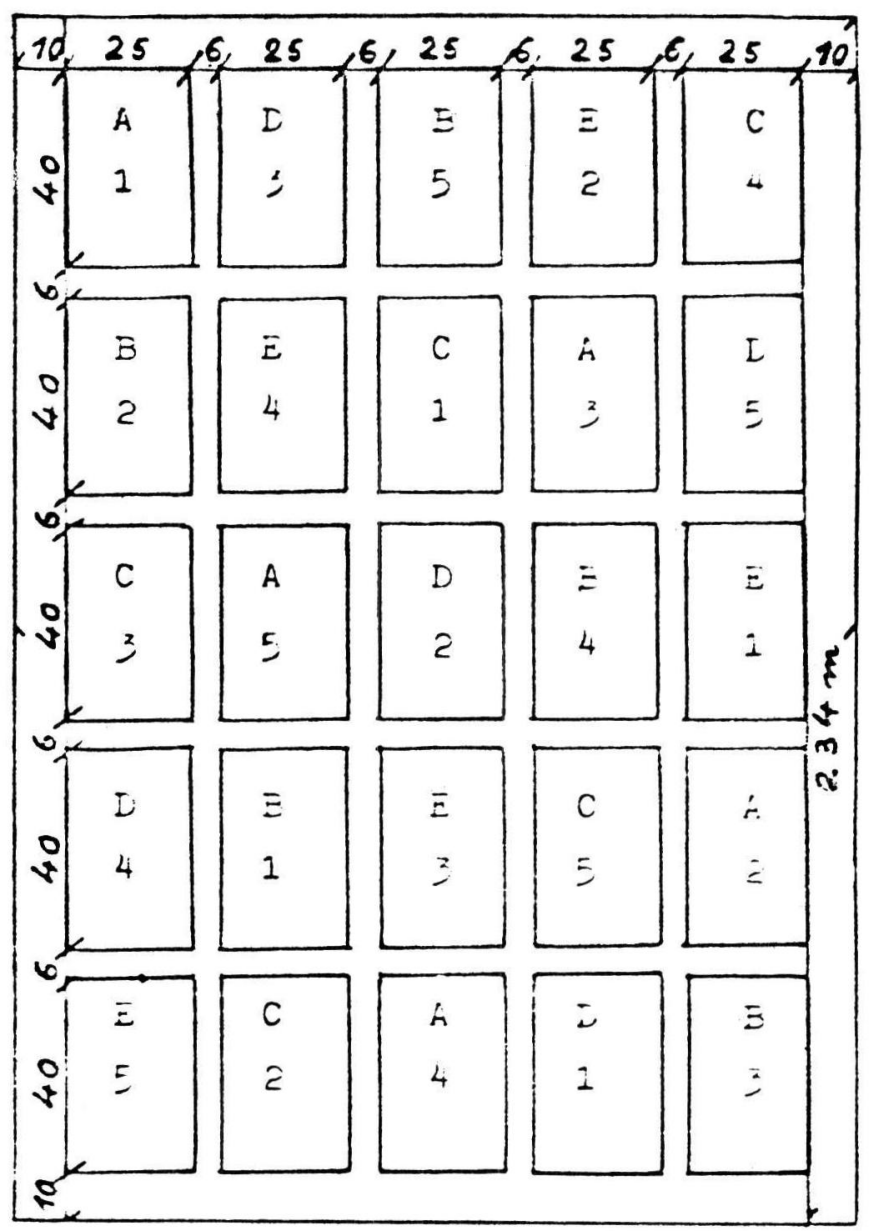

FIG. 1. - Dispositif expérimental de Lamotte-Beuvron

Intensité 1 : aucune éclaircie,

2 : la surface terrière est égale à 120 p. 100 de la référence,

3 : référence,

4 : surface terrière égale à 80 p. 100 de la référence,

5 : surface terrière égale à 70 p. 100 de la référence,

(L'intensité 1 représente environ 130 p. 100 de la surface terrière de référence.)

\section{Experimental design}

Thinning weight 1 : null ; (basal area $=1.3 \mathrm{G}$ ),

$2:$ basal area $=1.2 \mathrm{G}$,
$3: \mathrm{G}$,
$4:$ basal area $=0.8 \mathrm{G}$,
$5:$ basal area $=0.7 \mathrm{G}$.

Thinning method $A$ : thinning from below,

$B$ : thinning from above,

$C$ : free thinning,

$D$ : mixed thinning,

E : mechanical thinning (line thinning). 
Vingt-cinq placettes de 10 ares, séparées par des zones neutres ont été installées en 1964-1965 (fig. 1) ; dans chacune de ces placettes, 30 arbres de place, bien répartis sur le terrain, ont été sélectionnés ; cela correspond à un peuplement final de 300 tiges par ha.

Pour mettre en évidence l'effet $a$ ) de la densité, 5 intensités d'éclaircie ont été définies : pour l'intensité moyenne (intensité $n^{\circ} 3$ ), on suit la table de production de Décourt (1965). L'intensité no 2 est plus faible et les densités 4 et 5 plus fortes ; le critère de densité retenu est la surface terrière, somme des surfaces des sections des arbres à $1,30 \mathrm{~m}$ au-dessus du sol.

Les intensités d'éclaircies étant définies, il faut encore choisir les arbres à enlever : 5 modalités d'éclaircies ont été expérimentées pour tenter de mettre en évidence les autres causes de la compétition.

Pour mettre en évidence l'effet $c$ ) de la concurrence entre strates, les arbres sélectionnés étant des arbres dominants, on a distingué :

- la modalité A : éclaircie «systématique par le bas », où l'on retire les arbres les plus petits jusqu'à ce qu'on ait atteint la surface terrière fixée,

- la modalité B : éclaircie "systématique par le haut», où mises à part les 30 tiges sélectionnées, on retire les arbres les plus gros jusqu'à atteindre la surface terrière fixée.

Pour mettre en évidence l'effet $b$ ) de densité locale, on a distingué :

- la modalité $C$, dite « au profit des arbres de place » qui consiste à prélever la quantité d'arbres fixés uniquement autour des 30 arbres sélectionnés,

- la modalité $D$, éclaircie mixte classique,

- la modalité E, éclaircie par rangées tirées au hasard, et donc indépendamment de la situation des arbres de place.

Ces 5 modalités croisées avec les 5 intensités d'éclaircies conduisent donc en fait à un carré gréco-latin $5 \times 5$.

Mise en place de l'expérience.

Pour éviter les chablis, principalement dans les éclaircies fortes et dans la modalité $\mathrm{E}$ par rangées, les arbres ont été enlevés progressivement en plusieurs étapes ; des éclaircies ont été marquées en 1966, 1969,1972 et 1975. En ce qui concerne la modalité $E$, les risques de dégâts de vent étant importants, ce sont en réalité des demi-rangées qui ont été tirées au hasard ; lorsqu'un arbre de place se trouvait sur une de ces demi-rangées, il a bien entendu été conservé. Ces précautions indispensables permettent en 1976 de n'observer aucune disparition accidentelle d'arbre.

\section{3. - Premiers résultats}

Le tableau 1 donne les principales mesures faites en 1969 et 1975. 
TABLEAU 1

\begin{tabular}{|c|c|c|c|c|c|c|c|c|c|c|c|}
\hline \multirow{2}{*}{ Eclaircie } & & \multicolumn{2}{|c|}{$\begin{array}{c}\text { A } \\
\text { par le bas }\end{array}$} & \multicolumn{2}{|c|}{$\begin{array}{c}\text { B } \\
\text { par le haut }\end{array}$} & \multicolumn{2}{|c|}{$\underset{\text { avenir }}{C}$} & \multicolumn{2}{|c|}{$\begin{array}{c}\text { D } \\
\text { classique }\end{array}$} & \multicolumn{2}{|c|}{$\begin{array}{c}E \\
\text { en lignes }\end{array}$} \\
\hline & & 1969 & 1975 & 1969 & 1975 & 1969 & 1975 & 1969 & 1975 & 1969 & 1975 \\
\hline $\begin{array}{c}1 \\
\text { Témoin }\end{array}$ & $\begin{array}{l}\mathrm{N} / \mathrm{ha} \\
\mathrm{G} / \mathrm{ha} \\
\mathrm{Cg} \\
\mathrm{Ca} \\
\mathrm{Ha}\end{array}$ & $\begin{array}{l}2790 \\
22.8 \\
32 \\
48.5 \\
10.3\end{array}$ & $\begin{array}{c}2400 \\
29.0 \\
39.0 \\
55.1 \\
12.8\end{array}$ & $\begin{array}{l}3330 \\
27.2 \\
32 \\
45.9 \\
10.0\end{array}$ & \begin{tabular}{|l|}
2980 \\
34.0 \\
37.9 \\
52.1 \\
12.4
\end{tabular} & $\begin{array}{l}3270 \\
27.3 \\
32 \\
45.7 \\
10.7\end{array}$ & $\begin{array}{l}2790 \\
33.5 \\
38.8 \\
51.8 \\
13.2\end{array}$ & $\begin{array}{l}2650 \\
26.6 \\
36 \\
51.5 \\
11.4\end{array}$ & $\begin{array}{c}2220 \\
32.6 \\
42.9 \\
58.4 \\
13.8\end{array}$ & $\begin{array}{l}3290 \\
29.4 \\
33 \\
50.2 \\
11.0\end{array}$ & $\begin{array}{l}2760 \\
35.2 \\
40.0 \\
56.5 \\
13.4\end{array}$ \\
\hline $\begin{array}{c}2 \\
\text { Faible }\end{array}$ & $\begin{array}{l}\mathrm{N} / \mathrm{ha} \\
\mathrm{G} / \mathrm{ha} \\
\mathrm{Cg} \\
\mathrm{Ca} \\
\mathrm{Ha}\end{array}$ & $\begin{array}{l}3570 \\
28.8 \\
32 \\
46.0 \\
10.7\end{array}$ & \begin{tabular}{|l|}
2160 \\
31.1 \\
42.5 \\
52.6 \\
13.2
\end{tabular} & $\begin{array}{l}3410 \\
24.2 \\
30 \\
48.4 \\
10.2\end{array}$ & \begin{tabular}{|c|}
2770 \\
28.8 \\
36.2 \\
55.6 \\
12.8
\end{tabular} & $\begin{array}{l}2930 \\
24.7 \\
33 \\
47.5 \\
10.0\end{array}$ & $\begin{array}{l}2180 \\
28.0 \\
40.2 \\
54.7 \\
12.4\end{array}$ & $\begin{array}{l}3280 \\
28.1 \\
33 \\
48.6 \\
10.3\end{array}$ & $\begin{array}{l}2170 \\
31.1 \\
42.4 \\
52.8 \\
12.7\end{array}$ & $\begin{array}{l}2300 \\
23.4 \\
36 \\
54.9 \\
10.2\end{array}$ & $\begin{array}{l}1910 \\
30.2 \\
44.6 \\
62.2 \\
12.7\end{array}$ \\
\hline $\begin{array}{c}3 \\
\text { Moyenne }\end{array}$ & $\begin{array}{l}\mathrm{N} / \mathrm{ha} \\
\mathrm{G} / \mathrm{ha} \\
\mathrm{Cg} \\
\mathrm{Ca} \\
\mathrm{Ha}\end{array}$ & $\begin{array}{l}2260 \\
21.4 \\
34 \\
52.3 \\
9.8\end{array}$ & $\begin{array}{c}1510 \\
26.3 \\
46.8 \\
60.5 \\
12.2\end{array}$ & $\begin{array}{l}2700 \\
25.7 \\
35 \\
52.6 \\
10.7\end{array}$ & \begin{tabular}{c|}
1890 \\
22.8 \\
38.9 \\
61.5 \\
13.1
\end{tabular} & $\begin{array}{l}3400 \\
26.8 \\
31 \\
47.8 \\
10.2\end{array}$ & $\begin{array}{l}2100 \\
26.4 \\
39.8 \\
56.6 \\
12.7\end{array}$ & $\begin{array}{l}2900 \\
23.6 \\
32 \\
53.6 \\
10.0\end{array}$ & $\begin{array}{l}1570 \\
25.5 \\
45.2 \\
61.2 \\
12.7\end{array}$ & \begin{tabular}{|l}
3440 \\
27.7 \\
32 \\
45.6 \\
10.1
\end{tabular} & $\begin{array}{l}2160 \\
26.9 \\
39.6 \\
53.2 \\
12.5\end{array}$ \\
\hline $\begin{array}{c}4 \\
\text { Forte }\end{array}$ & $\begin{array}{l}\mathrm{N} / \mathrm{ha} \\
\mathrm{G} / \mathrm{ha} \\
\mathrm{Cg} \\
\mathrm{Ca} \\
\mathrm{Ha}\end{array}$ & $\begin{array}{l}2800 \\
25.3 \\
34 \\
48.3 \\
10.6\end{array}$ & $\begin{array}{c}1070 \\
22.6 \\
51.5 \\
57.6 \\
13.2\end{array}$ & $\begin{array}{l}3370 \\
26.2 \\
31 \\
47.0 \\
10.1\end{array}$ & $\begin{array}{c}1940 \\
22.2 \\
37.9 \\
56.3 \\
12.5\end{array}$ & $\begin{array}{l}2690 \\
23.3 \\
33 \\
49.9 \\
10.0\end{array}$ & $\begin{array}{l}1300 \\
21.5 \\
45.6 \\
60.6 \\
12.5\end{array}$ & $\begin{array}{l}2890 \\
26.1 \\
34 \\
51.9 \\
10.5\end{array}$ & $\begin{array}{c}1170 \\
21.4 \\
48.0 \\
62.1 \\
12.8\end{array}$ & $\begin{array}{l}3060 \\
24.7 \\
32 \\
49.7 \\
10.4\end{array}$ & $\begin{array}{l}1420 \\
21.1 \\
43.2 \\
59.4 \\
12.9\end{array}$ \\
\hline $\begin{array}{c}5 \\
\text { Très forte }\end{array}$ & $\begin{array}{l}\mathrm{N} / \mathrm{ha} \\
\mathrm{G} / \mathrm{ha} \\
\mathrm{Cg} \\
\mathrm{Ca} \\
\mathrm{Ha}\end{array}$ & $\begin{array}{l}3200 \\
27.9 \\
33 \\
48.8 \\
10.0\end{array}$ & $\begin{array}{l}740 \\
18.6 \\
56.2 \\
59.0 \\
12.4\end{array}$ & $\begin{array}{l}2410 \\
23.4 \\
35 \\
53.8 \\
10.4\end{array}$ & \begin{tabular}{|c|}
1410 \\
19.7 \\
41.9 \\
64.2 \\
13.1
\end{tabular} & $\begin{array}{l}3220 \\
25.8 \\
32 \\
46.8 \\
10.2\end{array}$ & $\begin{array}{c}1420 \\
19.0 \\
41.0 \\
56.9 \\
12.6\end{array}$ & $\begin{array}{l}2590 \\
24.3 \\
34 \\
48.8 \\
10.0\end{array}$ & $\begin{array}{c}1060 \\
19.7 \\
48.4 \\
57.5 \\
12.5\end{array}$ & $\begin{array}{l}3220 \\
27.6 \\
33 \\
45.4 \\
10.6\end{array}$ & $\begin{array}{c}1320 \\
18.8 \\
42.3 \\
55.9 \\
12.9\end{array}$ \\
\hline
\end{tabular}

Nombre de tiges par hectare: $N / h a$.

Surface terrière en $\mathrm{m}^{2} / \mathrm{ha}: \mathrm{G} / \mathrm{ha}$.

Circonférence de l'arbre de surface terrière moyenne: $\mathrm{Gg}$.

Circonférence de l'arbre de place de surface terrière. moyenne : $\mathrm{Ca}$.

Hauteur moyenne des arbres de place : Ha.

$\mathrm{N} / \mathrm{ha}=$ number of trees par hectare.

$\mathrm{G} / \mathrm{ha}=$ basal area in $\mathrm{m}^{2} / \mathrm{ha}$.

$\mathrm{Cg}=$ girth of the tree of mean basal area.

$\mathrm{Ca}=$ girth of the elite tree of mean basal area.

ha $=$ mean height of elite trees.

\section{4. - Discussion}

Des premières observations sont possibles sur l'accroissement relatif en surface terrière des 30 arbres sélectionnés dans chaque placette :

$$
\frac{\Delta G}{G}=\frac{(G / h a) 1975-(G / h a) 1969}{(G / h a) 1969} .
$$


Les valeurs trouvées figurent dans les tableaux 2 et 3 sous deux présentations :

TABLEAU 2

Accroissements relatifs suivant le plan du dispositif

Relative increments according to experimental design

$\begin{array}{ccccc}\text { A 1 } & \text { D 3 } & \text { B 5 } & \text { E 2 } & \text { C 4 } \\ 0,276 & 0,289 & 0,410 & 0,260 & 0,456 \\ \text { B 2 } & \text { E 4 } & \text { C 1 } & \text { A 3 } & \text { D 5 } \\ 0,306 & 0,406 & 0,273 & 0,309 & 0,379 \\ \text { C 3 } & \text { A 5 } & \text { D 2 } & \text { B 4 } & \text { E 1 } \\ 0,389 & 0,410 & 0,288 & 0,419 & 0,248 \\ \text { D 4 } & \text { B 1 } & \text { E 3 } & \text { C 5 } & \text { A 2 } \\ 0,414 & 0,270 & 0,338 & 0,466 & 0,299 \\ \text { E 5 } & \text { C 2 } & \text { A 4 } & \text { D 1 } & \text { B 3 } \\ 0,494 & 0,305 & 0,393 & 0,272 & 0,342\end{array}$

TABLEAU 3

Accroissements relatifs classés par intensités et modalités d'éclaircie

Relative increments according to thinning weights and thinning methods

$\begin{array}{ccccc}\text { A 1 } & \text { B 1 } & \text { C 1 } & \text { D 1 } & \text { E 1 } \\ 0,276 & 0,270 & 0,273 & 0,272 & 0,248 \\ \text { A 2 } & \text { B 2 } & \text { C 2 } & \text { D 2 } & \text { E 2 } \\ 0,299 & 0,306 & 0,305 & 0,288 & 0,260 \\ \text { A 3 } & \text { B 3 } & \text { C 3 } & \text { D 3 } & \text { E 3 } \\ 0,309 & 0,342 & 0,389 & 0,289 & 0,338 \\ \text { A 4 } & \text { B 4 } & \text { C 4 } & \text { D 4 } & \text { E 4 } \\ 0,393 & 0,419 & 0,456 & 0,414 & 0,406 \\ \text { A 5 } & \text { B 5 } & \text { C 5 } & \text { D 5 } & \text { E 5 } \\ 0,410 & 0,410 & 0,466 & 0,379 & 0,494\end{array}$

La mesure de $\Delta G / G$ prise comme indicateur de la compétition a l'avantage d'éliminer des différences éventuelles de productivité d'une placette à l'autre ; on observe en particulier que pour les 5 placettes A1, B1, C1, D1, E1 d'intensité d'éclaircie nulle, et qui ont par conséquent subi le même traitement, les 5 valeurs trouvées sont très voisines : $0,276,0,270,0,273,0,272,0,248$.

L'analyse de variance a donné les résultats suivants :

- Au niveau du dispositif : il n'y a pas de différence significative au seuil de 5 p. 100 entre les lignes et entre les colonnes (la différence entre les colonnes n'est significative qu'au seuil de 25 p. 100) ;

- Au niveau des traitements : il y a une différence très significative entre les intensités d'éclaircies ; ceci confirme ce qui est déjà largement connu : plus les éclaircies sont fortes, plus les arbres restants ont un accroissement fort, quelle que soit la manière d'enlever les arbres. Cependant, étant donné le jeune âge du peuplement et la faible durée de l'expérience, ce résultat est déjà très important. La faible durée de l'expérience empêche encore d'étudier d'éventuelles pertes de production dans les éclaircies fortes.

- Par contre, la différence entre les modalités d'éclaircies n'est significative qu'au seuil de 7 p. 100 ; les traitements $B$ et $C$ donnent des accroissements relatifs légèrement supérieurs respectivement à $A$ et $(D+E)$ ce qui semblerait confirmer les hypothèses de départ :

- les arbres dominés ne concurrencent pas les arbres dominants (traitements $A$ et $B$ ).

- la concurrence des voisins immédiats est plus forte que celle des arbres plus éloignés (traitements $C$ et $D+E$ ).

Mais les différences ne sont pas encore suffisamment probantes pour qu'on puisse donner des indications sylvicoles précises ; ce qui est très probable, c'est que dans 
l'optique choisie d'une sylviculture d'arbres de place, l'éclaircie par le bas (A), l'éclaircie mixte classique (de type $D$ ), ef l'éclaircie par rangées $(E)$ ne donneront jamais des résultats meilleurs que des éclaircies par le haut (B) ou concentrées autour des arbres de place (C).

Il est nécessaire de poursuivre cette expérience pour confirmer les premiers résultats. Mais parallèlement, il faudrait entreprendre d'autres expériences, soit dans des plantations régulières pour mettre en évidence l'influence de la variabilité génétique sur la concurrence, soit dans des dispositifs mono-clonaux pour mettre en évidence l'effet local de la densité au voisinage de l'arbre, soit dans des mélanges à 2 clones pour mettre en évidence l'influence du mélange.

Enfin, mais c'était prématuré ici, il serait nécessaire d'affiner l'analyse statistique par une modélisation au niveau de l'arbre et de ne pas se contenter de l'analyse sur $\Delta G / G$ qui n'est qu'un indice global au niveau du peuplement, et qui ne prend pas en compte la distribution spatiale des arbres de place et des arbres enlevés en éclaircie.

\title{
Conclusion - Résumé
}

L'effet de la concurrence en forêt est quelquefois malaisé à mettre en évidence parce qu'il est masqué par d'autres facteurs. Une expérience faite dans un peuplement de pin sylvestre a confirmé l'effet important de la densité moyenne des peuplements sur l'accroissement des arbres restants ; par contre, il est encore prématuré de vouloir tirer des informations sur la concurrence entre strates et sur les effets de la densité locale.

Reçu pour publication en avril 1977.

\section{Summary \\ Considerations on the first results of a thinning experiment in scotch pine in the forest of Lamotte-Beuvron}

\begin{abstract}
Competition between trees in forest stands is sometimes difficult to prove due to the great number of factors which influence the growth. In this experiment we could confirm the effect of mean density of a stand upon the increment. But, at the present stage, neither the local density nor the competition between strata appeared to have an effect upon the basal area increment of elite trees.
\end{abstract}

\section{Références bibliographiques}

DECOURT N., 1965. Les tables de production pour le Pin sylvestre et le Pin laricio de Corse en Sologne. Revue Forestière Française, 1965, 17 (12), 818-831.

DECOURT N. et LEMOINE M., 1974. Premiers résultats d'une expérience clinale d'espacement avec Populus trichocarpa. Ann. Sci. For., 31 (3), 171-180.

ILLY G. et LEMOINE B., 1970. Densité de peuplement, concurrence et coopération chez le pin maritime. 1. Premiers résultats d'une plantation à espacement variable. Ann. Sci. For., 1970, 27 (2), 127-155.

JACQUARD P., 1975. Concurrence intraspécifique et potentialité de rendement. Ann. Amélior. Plantes, 1975, 25 (1), 1-24.

LEARY; R. A., 1970. Nonlinear functional equation models of forest dynamics. Seminar, Feb. 18, 1970, N. C. For Exp. Sta. St-Paul Minn, 21 p.

DE WIT C. T., 1960. On competition. Versl. Landbouwk. Onderz, no 66, 8, 82 p. 